\title{
Meteoroid streams: mathematical modelling and observations
}

\author{
Galina O. Ryabova ${ }^{1}$ \\ ${ }^{1}$ Research Institute of Applied Mathematics and Mechanics of Tomsk State University, Tomsk, \\ 634050, Russia \\ email: ryabova@niipmm.tsu.ru
}

\begin{abstract}
Mathematical modelling of meteoroid streams formation and evolution is a very fruitfull method for obtaining not only cosmogonical knowledges, but information about the stream up-to-date structure as well. In this review we consider advances of the method, and applications to meteoroid streams of different schemes of formation. We also discuss the part played by observations, and feedback between models and observations. Attention is also drawn to some unresolved problems and promising areas of application.
\end{abstract}

Keywords. meteors, meteoroids, comets: general, asteroids, methods: numerical, methods: statistical

\section{Introduction}

Computer simulation of meteoroid streams formation and evolution has gone through several stages, which were dictated mainly by computer power. At the first stage mainly a single orbit was integrated (the mean orbit of a shower $\dagger$ or the orbit of its parent comet, e.g. Weiss (1868)). Or it was not numerically integrated, only the secular perturbation changes were calculated (e.g. Plavec 1950). Or one orbit was integrated, and dispersion of the meteoroid elements was calculated analytically (e.g. Hamid 1951). Or it was a pure analytical method entailed by cumbersome mathematical manipulations (e.g. Murray 1982). Results concerned mainly such problems as the origin of a meteoroid stream, the visibility conditions of a meteor shower, displacement of the shower's node etc.

At the second stage general dynamics of streams was studied by integrating of small amounts of meteoroid orbits (e.g. Kazimirčak-Polonskaya, Belyaev, Astapovič \& Terent'eva 1968; Kazimirchak-Polonskaya, Belyaev \& Terent'eva 1972; Williams, Murray \& Hughes 1979).

The real "ideological" break through has happened after Fox, Williams \& Hughes (1983) work. For one thing, for the first time there were obtained model rate profiles of a shower (the Geminids), for another, an idea about approximation of changes in orbital elements by power series of time was new, and, as it turned out later, very fruitful (see Section 5).

With computer power increasing, large scale integration studies have became possible. McIntosh \& Jones (1988) modelled the Comet Halley meteoroid streams, Ryabova (1989) modelled the Geminid stream, Brown \& Jones (1998) modelled the Perseid stream, Ryabova (2001a) modelled the meteoroid streams of asteroid (1620) Geographos, Vaubaillon, Colas \& Jorda (2005b) modelled the Leonid meteoroid stream etc.

The objective to be pursued by the present work is not the historical review of mathematical modelling of meteoroid streams. We shall discuss the general principles

$\dagger$ This is not misprint. The mean orbit of the stream is not the same, as the mean orbit of the part of the stream registered at the Earth. 
of mathematical modelling (Section 2), and in particular methods of calculations of the stream's age (Section 3) and the ejection velocity (Section 4). At present we have only two more or less full models for meteoroid streams, namely for the Geminid and the Perseid meteoroid streams. These models will be discussed in Sections 5 and 7. In Section 8 we shall consider a model aimed at the Leonids predictions, but having all the potential to grow into full model. The special attention will be called to observations as both base for a model and criterion for its validity. Origin of the Geminid-type streams is still an open question, it is discussed in Section 6. The last Section 9 outlines some results showing that mean motion resonances can cause evident changes in the structure of a meteoroid stream.

\section{Mathematical modelling. General}

The process of mathematical modelling could be subdivided into four stages.

\subsection{Compiling of physical model for meteoroid stream and cometary decay}

We should know the main structural parameters of a stream and interrelations among them. These parameters are

- period of the visibility of the stream on the Earth;

- outbursts (time, location);

- node regression or progression;

- twin showers, if exist;

- flux density profile of the meteor shower;

- profile of mass distribution index $s$;

- activity variations along the orbit (or, what is the same, from year to year)

- "windage" of meteoroids, i.e. $A / m$, where $m$ and $A$ are the mass of the meteoroid and its cross-sectional area, respectively;

- orbital parameters for meteoroids of different masses;

- radiant (location, motion, configuration);

- grouping meteoroids in the stream;

- flickering of meteors light-curve;

- space distribution of meteoroids.

The parameters listed not in importance. As it will be discussed later, even for the most studied streams uncertainty for some of the parameters is too lagre.

It is very important to know the parent body for a meteoroid stream, because the model constructed on the basis of the shower mean orbit (i.e. on the mean orbit of the meteoroids registered at the Earth) cannot give correct spatial distribution of the meteoroid stream matter. If the parent body is known, the very first and key parameter we should determine is age of the meteoroid stream. Let us define "age" as age of meteoroids. If the parent comet have been active for a long time, the stream contains meteoroids of different age. But sometimes a stream can be generated on relatively short time interval, so its meteoroids have approximately the same age. The narrow, practical meaning of this term is "the date from which we begin our modelling". To model decay of the comet we should know the following parameters:

- ephemeris of the comet;

- dust production rate;

- distribution of ejection velocity vectors in magnitude and direction. 


\subsection{Choise of mathematical methods}

In the overwhelming majority of cases under the term "model of a meteoroid stream" we imply an ensemble of meteoroids, whose ejections were modelled and evolution followed. So, first of all this is a choise of methods for calculation of the orbital evolution.

In not so remote past the Gauss-Halphen-Goryachev method was used for integration of orbits very intensively, because it is very fast method. With this method only secular perturbations of the first order can be calculated. So, it cannot process correctly close encounters of meteoroids with planets, or resonance cases, therefore, the validity of its application should be carefully verified. For the Geminid meteoroid stream it works perfectly. Moreover, the Geminid stream is so "unperturbed", that we may use polynomial approximation instead of direct integration (see Section 5).

Now, when computing power allows for large scale integration studies, the problem comes to choosing an integrator and algorithm for the most part. There are a number of publications considering algorithms for high-accuracy long-term simulation of the small bodies motion (e.g. Bordovitsyna, Avdyushev \& Chernitsov 2001). Numerical methods of integration of the motion equations are applicable to every kind of motion. But they are expensive (even now), and have time limitations because of the accumulation of errors.

To study long-term evolution a symplectic integrator may be applied (Brown \& Jones 1998).

It is worth to mention a method developed by Babadzhanov \& Obrubov (1987) both in the historical context, and because their results are still referred to. The metod use the following quasi-integrals of motion:

$$
\begin{gathered}
\left(1-e^{2}\right) \cos ^{2} i \approx \text { const }, \\
e^{2}\left(0.4-\sin ^{2} i \sin ^{2} \omega\right) \approx \text { const } .
\end{gathered}
$$

The first integral was found by Moiseev (1945), and the second one by Lidov (1961). Assuming the longitudes of perihelion of orbits of all the meteoroids to be constant, i.e. $\Omega+\omega=\pi=$ const, and assuming that a stream lives long enough that the argument of perihelion makes a full revolution $\left(0^{\circ}-360^{\circ}\right)$, we may outline a surface, enclosing all possible meteoroid orbits. Babadzhanov \& Obrubov have shown that a meteoroid stream can produce up to 8 meteor showers. My own rough estimate of the time for complete $\omega$ circulation is: 16 thousands years for the Quadrantid stream (assuming asteroid 2003EH1 being its parent body), 37 thousand years for the Geminid stream, and $>1 \mathrm{mln}$ years for the Perseid stream. So this method is not applicable to the Geminid meteoroid stream, because its age is about two thousands years (Ryabova 1999).

It is important to define exactly which forces are included in the model. What major planets should be taken into account in the calculations of gravitational perturbations, what radiational perturbations, etc.

A stream model should be statistically relevant, i.e. the results should not fluctuate with increasing number of modelled meteoroids. From the other side, integration of millions of orbits is rather expensive. So, a known method of weighting coefficients could be applied in some cases. The technique consists in ascribing to every model meteoroid a definite weight depending, as a rule, on dust production rate.

\subsection{Comparison with observations and constraining the model}

Parameters, which can be obtained from a stream model to compare with observations are listed in Subsection 2.1. We shall discuss these parameters in details on concrete examples in sections considering specific meteoroid streams. 
It is clear, that if there is an agreement between the model and observations within the level of errors, it is a good argument in favour of the model reliability. Sometimes we could try to solve an inverse problem, i.e. to constrain initial model parameters in such a way, that resulting parameters agree with experimental ones.

\subsection{Revision of the model}

Even the most perfect model of its time has limitations determined by the level of experimental data, and, in a more comprehensive sense, by the level of knowledge. A time inevitably comes, when the model should be "upgraded", or the new model should be constructed.

One practical conclusion: referring to 30 -years old results of modeling one should be very careful.

\section{Age of meteoroids}

The first review of the meteoroid streams age determination was, according to my knowledge, published by Plavec (1955a). Some of the methods described in the work lost their validity. For example, now we know that if a stream is not distributed around all the orbit, it is not necessarily young (Williams 1997). Some others as, for example, the evaluation of the upper limit of the age as lifetime for the largest meteoroids in the stream, still can be used.

A detailed analytical review of the methods for determining the age of a meteoroid stream as applied to the Geminid stream could be found in (Ryabova 1999). The main points from the review will be abstracted below. But the Geminid meteoroid stream seems to be a stream generated during a relatively short period of time. The streams, replenished by new portions of meteoroids for a long time, as, for example, the Leonid or the Perseid streams should be treated differently.

\subsection{Retrospective analysis of evolution}

Since stream meteoroids and their parent bodies had been once a single whole, an attempt to trace the orbital evolution of the parent body and meteoroids back in time and find moment of their separation suggests itself. But this problem cannot be solved unambiguously and with sufficient accuracy, because of low accuracy of the measured orbital parameters, and uncertainties in physical parameters of meteoroids.

\subsection{Mass segregation}

The rate of change in orbital elements of meteoroids, in particular, the semimajor axis and the eccentricity depends on the meteoroids windage $(A / m)$. The Poynting-Robertson (PR) effect and its corpuscular analogue (Ryabova 2005) provide the most significant contribution to the phenomenon.

The following equation can be written for the semimajor axis of a meteoroid:

$$
a=a_{c 0}+\Delta a_{0}+(\Delta a)_{g r}+(\Delta a)_{P R}+\varepsilon a,
$$

where $a_{c 0}$ is the semimajor axis of the parent body orbit at the time of ejection, $\Delta a_{0}$ is the increment due to the ejection velocity and light pressure; $(\Delta a)_{g r},(\Delta a)_{P R}$, and $\varepsilon a$ are the increments of the semimajor axis due to gravitational perturbations, PR-effect (plus its corpuscular analogue), and all other factors, respectively. The contributions from every term are considered in (Ryabova 1999).

In practice, when estimating age, equation (3.1) simplifies to

$$
a-a_{c} \approx(\Delta a)_{P R},
$$


where $a_{c}$ is the semimajor axis of the parent body at present time. Ryabova (1999) has shown that such approach leeds to unacceptably large errors. But if the age is estimated from the mean orbits of meteoroids belonging to close mass ranges, the error in age estimation decreases considerably.

\subsection{Mathematical simulation}

If structural characteristics of the observed and simulated streams are in agreement, this is a good argument in favour of the age of the stream assumed by the model. The reverse is true only partially, because the model is defined by many parameters, as important as the age itself but, unfortunately, not known better than the age. As an illustration the models for the Geminid meteoroid stream are compared for ages 2000 and 10000 years in (Ryabova 1999).

Brown \& Jones (1998) (their model is described in Section 7) tried to estimate the Perseid stream age comparing model and observed structural characteristics of the shower. From the model the authors estimated the "average" radiant dispersion with time, shift in position of the radiant with time, shift in the position of the main visual maximum with time, width of the ZHR profile, and the full nodal spread of the current shower. The estimates range are in the interval $(7-180) \times 10^{3} \mathrm{yr}$. The dispersion is rather large, and the reason is primarily accuracy and quality of observations. The limitations of the model are in second place.

For streams like the Perseid and Leonid streams, where the parent comet have been active for a long time, it is possible to obtain time of meteoroids ejection from comparing model and observed position and strength of the definite shower's maximum or outburst (e.g. Brown \& Jones 1998; Asher, Bailey \& Emel'yanenko 1999; Asher 1999).

\subsection{Meteoroids lifetime}

In the interplanetary space meteoroids are subject to destruction from many factors. Estimations of the meteoroid lifetime were improved over the time (Whipple 1967a; Whipple 1967b; Dohnanyi 1978; Tokhtas'ev 1982; Leinert, Röser \& Buitrago 1983; Olsson-Steel 1986; Steel \& Elford 1986). In all cases it was confirmed that, for particles with mass $\geqslant 10^{-6}-10^{-7} \mathrm{~g}$, catastrophic collisions with sporadic meteoroids prevail. The PR-effect plays a secondary role. Comparison between the meteoroid lifetime and the mass distribution of the shower meteoroids allows sometimes make a conclusion on the stream age. For example, if there are no particles of a certain mass range in the stream, it can be suggested that they were removed by one of the destruction factors. The upper limit of the stream's age could be estimated as lifetime of largest meteoroids.

\subsection{Age changes in mass distribution}

An indirect estimate of six (Geminid, D.Arietid, Perseid, Quadrantid, $\eta$-Aquarid and $\xi$-Aquarid) meteoroid stream's age was obtained by Dohnanyi (1970), who studied the effect of impacts with sporadic background on the initial mass distribution in the stream. He revealed that five out of the six streams considered (exclusion is the Quadrantid stream) are sufficiently old that their small particles have reached a steady state distribution under the collisional environment of the sporadics, but at the same time they are sufficiently young that their large particles still have a distribution similar to stream's initial distribution. It is unknown, however, what the actual age that corresponds to the terms "sufficiently young" and "sufficiently old". It is not inconceivable that the described results, based on 35-years old data, need revising. 


\subsection{Spin-up time scale}

Beech (2002) suggested a potentially powerful method for finding the time since ejection of meteoroids from their parent body. The approach relies upon being able to estimate the pre-atmospheric spin rates of meteoroids from the effect of light-curve flickering. The basic idea is using the 'windmill effect', which relies on non-isotropic photon scattering by meteoroid surface irregularities to produce a non-zero net torque. For three Geminid meteoroids under consideration the implied age is between 1000 and 4000 yr. Certainly, this method needs further investigation.

\section{Velocity of ejection}

The first publications devoted to ejection theory of the meteoroid stream formation appear to be the ones by Plavec $(1955,1957)$. He derived formulae giving the changes of the orbital elements owing to the ejection in the framework of the two body problem. These formulae were used lately in many researches.

A recent review on the determination of the ejection velocity of meteoroids from cometary nuclei could be found in (Williams 2001). Below are some additions.

\subsection{Methods based on ejection theory}

A technique for calculation of the ejection velocity was developed by Babadzhanov, Zausaev \& Obrubov (1980). The equations of motion of a parent body and the mean orbit of a meteoroid stream (or an orbit of an individual meteoroid) are integrated backward numerically, and the minimum of the minimal distance between the orbits is computed. The point on the cometary orbit corresponding to this minimum is assumed to be the point of ejection. Taking into account that the cometary and the stream orbits generally are not intersecting, the ejection velocity vector can be determined only approximately in the following way. Let $\Delta a=a_{c}-a_{s}$ be the difference between the cometary and stream orbits at time of the ejection, $\delta a$ the difference between the cometary orbit and an ejected meteoroid orbit at the given ejection velocity vector, calculated from formulae by Plavec (1955), $\epsilon_{a}$ the dispersion in observed meteoroids semi-major axes. The ejection velocity vector $\boldsymbol{c}$ is tabulated in reasonable limits. If the inequality $|\delta a-\Delta a| \leqslant \varepsilon_{a}$ is fulfilled, the analogical inequalities for other orbital elements $(e, i, \Omega, \omega)$ is checked. In such a way, a vector $\boldsymbol{c}$ obeying the set of inequalities is considered as the ejection velocity vector. Later was found that errors in initial orbital elements are too large to determine the accurate time and point of ejection. And even if they were precise, the estimation of $\boldsymbol{c}$ from non-intersecting orbits is too rough.

For completeness, a mention should be made to determining of the ejection velocity from difference between the parent and meteoroid semi-major axes without consideration on the evolution. This method was used by Williams (1996) for six major streams. But these determination, as noted later by Arter \& Williams (2002), are not reliable for the same reason, namely, large errors in the observed meteoroid semi-major axes.

A current difference between the orbits of a parent body and the meteoroids of a stream is due to the ejection process and subsequent changes in orbital parameters due to gravitational and non-gravitational perturbations. One of the parameters, namely the longitude of the ascending node is determined much more accurately than others. So, it suggests itself for the ejection velocity determination.

Such an attempt was made by Andreev (1986, 1987, 1995) who derived equations for change in the orbital elements that arises from an impuls which a meteoroid gets during ejection. A dependence of the ejection velocity from the particle mass was also taken into 
account. The estimations made by this method are very rough, and can serve only as zero approximation because evolution process was not taken into account.

Another attempt to elaborate a method for the ejection velocities deducing from the observed orbital parameters of meteoroids was made by Ma \& Williams (2001). Using the expression for $\Delta \Omega$, which is analogous to the one by Andreev (1986), but was derived by Ma, He \& Williams (2001) independently, the authors found a special way for its application. To exclude influence of gravitational and non-gravitational perturbations, the method was applied to meteor outbursts, taking into account that in outbursts we are probably observing very young meteoroids. Ma \& Williams also use the fact that if meteoroids are ejected at the nodes, there is no change in $\Omega$. So, the node change $\Delta \Omega$ is interpreted as the difference between the node of the parent comet and the nodal position of the maximum activity in the outburst.

Resume: all the described above methods of the ejection velocity determination give no really reliable results.

\subsection{Comet Halley ejection velocities}

In modelling a meteoroid ejection we mainly use Whipple's formula (1951), which gives the cometocentric velocity of a particle dragged away from the surface of the cometary nucleus by the radial gas flow, or one of its modification (e.g. Hughes 2000). Crifo (1995) and Crifo \& Rodionov (1997) formulae are also used.

Could we check these theoretical formula somehow? An opportunity came when a lot of experimental data was obtained during the last approach of Comet Halley to the Sun in 1986. It should be noted that there were not immediate measurements of particles ejection velocities. Moreover, a detailed comparison of different estimates is difficult, because they refer to different particle's masses and different arcs of the cometary orbit. An approximate relation is the following: the ejection velocities obtained by the FinsonProbstein method are 1.5-2 times lower than those obtained from an analysis of the particle's flux measurements by the DUSMA dust-mass analyzers installed on Vega-1 and Vega-2 spacecrafts, and these estimations are in turn 1.5-2 times lower than the values obtained from Whipple's formula for comparable conditions. A detailed review can be found in (Ryabova 1997).

Most of the estimations are related to very small particles $\left(m<10^{-9} \mathrm{~g}\right)$. But Richter, Curdt \& Keller (1991) managed to calculate several trajectories of large particles at the last stage of the Giotto approach. As a result, the ejection velocities were estimated to be $\sim 40 \mathrm{~m} \mathrm{~s}^{-1}$ for particles with $m=0.001 \mathrm{~g}$ (the ejection time being about $6-9$ days prior to passage of perihelion, i.e. for true anomaly of the comet $v \approx-20^{\circ} \ldots-30^{\circ}$ ) and less than $10 \mathrm{~m} \mathrm{~s}^{-1}$ for particles with $m=0.01 \mathrm{~g}$ (supposly for $v \approx 0^{\circ}--25^{\circ}$ ). Let us make estimations according to the Whipple's formula: in the first case $c \approx 120-180 \mathrm{~m} \mathrm{~s}^{-1}$, in the second $-c \approx 80-120 \mathrm{~m} \mathrm{~s}^{-1}$ (estimations were made for spherical particles of density $\left.0.35 \mathrm{~g} \mathrm{~cm}^{-3}\right)$.

At the time of the Vega-1 approach to comet Halley, the onboard instrument "Photon" measured the flux of comparatively large dust particles $\left(m \geqslant 10^{-9} \mathrm{~g}\right)$. Ryabova (1997) proposed a probabilistic method that enables one to model the stream of particles ejected from a cometary nucleus into the spacecraft trajectory. The best agreement between the model and the experimental profiles was achieved when the mean ejection velocity was equal to about $8 \%$ of the velocity obtained from the Whipple's formula.

Resume: Further development of the theory of particle acceleration by the gas of the cometary atmosphere is needed. New experiments in situ conducted in future space missions, including direct measurements of the velocity of dust grains and the determination of their physical characteristics could give invaluable assistance in this research. 


\section{The Geminid meteoroid stream}

There were a number of the Geminid stream modelling, every of which laid a brick into contemporary understanding of the stream structure and process of formation. Though this work is not, as mention have been made of, an historical review, some of these modelling are direct predessors of the model discussed below, namely works by Fox, Williams \& Hughes (1982), Fox, Williams \& Hughes (1983), Jones (1985), and Jones \& Hawkes (1986). The last version of the Geminid meteoroid stream model was presented on IAU Coll. 197 by Ryabova, but it was not published in full (a breaf could be found in Ryabova (2004)). The previous version of the model could be found in Ryabova (2001b).

\subsection{Orbits}

The orbits of the Geminid meteoroid stream, as well as the one of the asteroid (3200) Phaethon (the Geminid's parent body) are located far inside the Jupiter's orbit (for Phaethon $a=1.27 \mathrm{AU}, e=0.9, i=22^{\circ}$ ). Numerical integration of the asteroid's orbit has shown, that its semimajor axis is practically constant (changes are in the third decimal place), and changes in other orbital elements are smooth. The same is true for the most part of the Geminid's meteoroids. So changes in orbital elements may be approximated by a set of nested polynomials of the form

$$
b=b_{0}+\sum_{j=1}^{n} \sum_{k=0}^{m} b_{j k} a_{0}^{k} t^{j},
$$

where $b$ is one of the Keplerian elements $(a, e, i, \Omega, \omega), t$ is the time from the initial moment, $b_{0}$ is the ininial value for an element (at $\left.t=0\right)$.

Because the radiation pressure and the PR-effect were taken into account, the polynomials, and, respectively, the models were constructed for particles of two masses $\left(m_{3}=2.14 \times 10^{-3} \mathrm{~g}\right.$ and $m_{4}=2.14 \times 10^{-4} \mathrm{~g}$, if particles are spherical with density of $\left.1 \mathrm{~g} \mathrm{~cm}^{-3}\right)$. Calculations of evolution for 10 millions of meteoroids take only several minutes.

\subsection{The stream's structure}

Here we consider only the cometary model of the stream formation, i.e. ejection points at the reference orbit are uniformely distributed along the true anomaly, that is approximately consistent with dust production rate proportional to $r^{-4}$, where $r$ is heliocentric distance. The ejection velocities have been determined using the Whipple (1951) formula, and their directions were distributed uniformely in the solar hemisphere. The stream's age was taken of 2000 years (Ryabova 1999). It was assumed that the stream was generated during a short period of time, maybe even during a single revolution.

In fig. 1 a part of the Geminid's model cross-section in the ecliptic plane for particles of two masses $m_{3}$ and $m_{4}$ is shown. Each of the two model differential streams $\nmid$ follows its own evolutionary path, because, firstly, influence of PR-effect and radiation pressure depends on $A / m$, and secondly, because the ejection velocity also depends on meteoroid's mass. That is why the densest parts of the streams, which at ejection moment $(t=0)$ coincided with the parent (reference) orbit, do not coincide now. At fig. 1 they are designated as "reference orbit $m_{3}$ " and "reference orbit $m_{4}$ ". The second, as it should be because of the PR-effect, is nearer to the Sun.

The shower activity profiles, i.e. flux density variations along the Earth's orbit is designated by $A$. Having the profiles for two meteoroids masses we can calculate a profile for

$\dagger$ Differential stream is a stream consisted of meteoroids of definite mass, e.g. $m_{3}$ or $m_{4}$. Cumulative stream consists of meteoroids with masses $m>m_{0}$. 


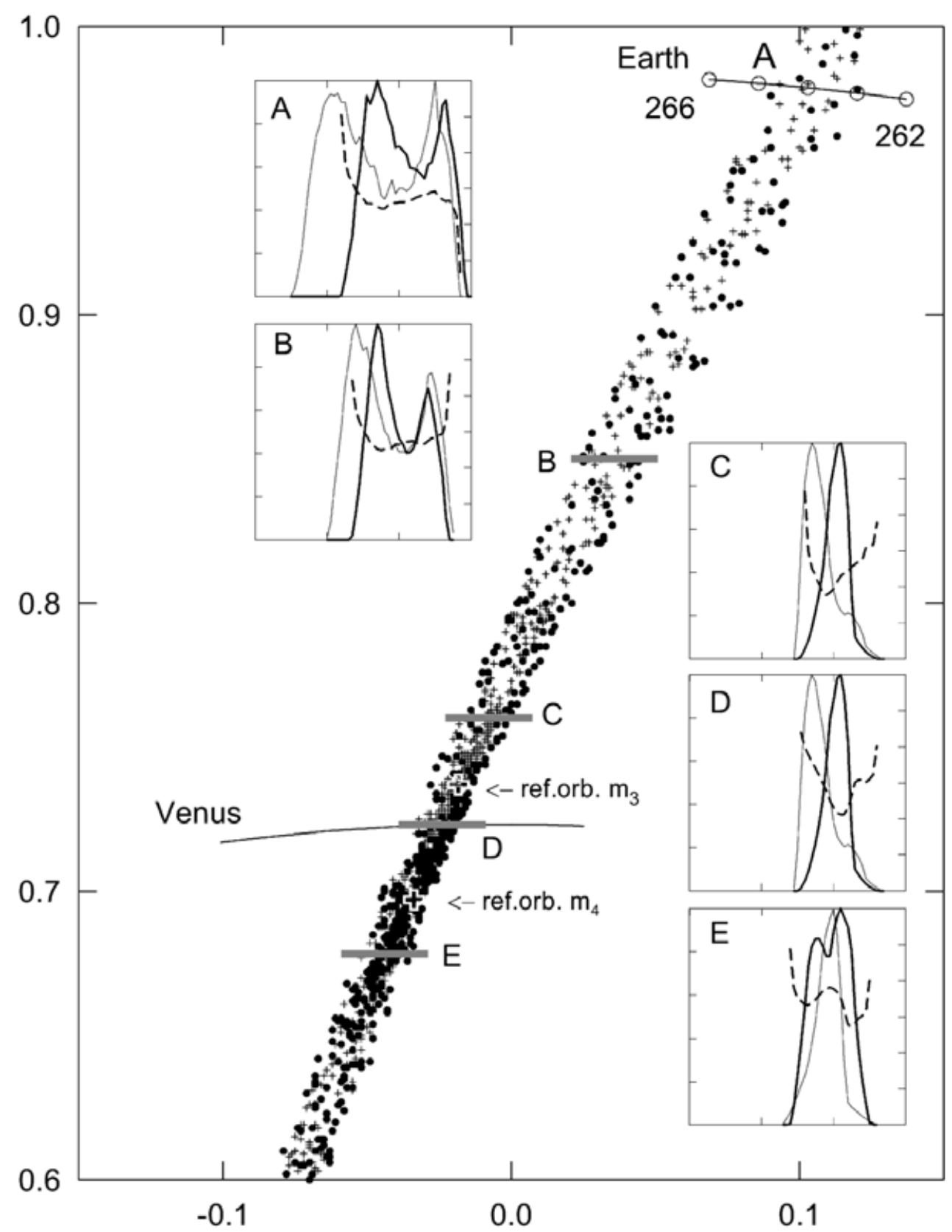

Figure 1. Geminid's model cross-section in the ecliptic plane for orbits of particles with masses $m_{3}(+)$ and $m_{4}(\bullet)$. The Earth's orbit in the interval $262^{\circ}-266^{\circ}$ in solar longitudes is designated by $A$. Other sections are designated by $B-E$. In the small panels, designated by $A-E$, activity profiles, i.e. flux density variations along the Earth's orbit, for particles masses $m_{3}$ (thick line) and $m_{4}$ (thin line), and a profile for mass index $s$ (dashed line) are shown. The profiles are calculated along the corresponding sections. 
mass index $s$. If the Earth's orbit intersects the model stream in other place, for example, along line $B$, the profile's shape will be different. The problem is that in reality we do not know where exactly the Earth crosses a model stream. To put it more precisely, we do not know the exact location of the stream cross-sections to the ecliptic plane. The main reason is that the moment of the stream generation is unknown. In this model the stream age is taken 2000 years. But if we take for example 2100 years, the stream's structure will be practically the same, but location of the cross-sections in the ecliptic plane will be shifted. Moreover, the accurate orbit of the parent body is not known, because the change of the cometary orbit during its trapping into the current orbit probably was considerable (see Section 6). Using approximations instead of numerical integration also shifts the location of the model stream. But we may use observations to fit or, better to say, to calibrate the model. The experimental profiles obtained by many-years of radar observations looks like something between $B$ and $C$ profiles (Bel'kovich 2005). To calibrate the model we should have flux density profiles with resolution of about $0.1^{\circ}$ in solar longitude, or better. Now the work on the flux profiles is underway.

Let us assume for the moment that the profiles $B$ are closest to the experimental ones, so the model stream should be moved in such a way that the Earth's orbit passed through the stream along the line designated by $B$. Then, knowing the particle's space density in the vicinity of the Earth's orbit, and using it as a reference one, we can evaluate quantitatively the particle's space density far from the Earth orbit. Moreover, we can evaluate the mass of the Geminid meteoroid stream.

It is very important that a model adjusting the flux density profile was used, and not a ZHR-profile (Zenith Hourly Rate) or simply a rate profile, because the rate profiles are not clarified from observational selection. The same is true in relation to the profile $s$. The profile obtained from hourly rates is burden by observational selection.

Besides factors of observational selection inherent to the various methods of observation (radar, visual etc.), there is astronomical selection, i.e. the alteration of the meteoroid flux in the gravitational field of a moving planet (Bel'kovich 1983; Bronshten 1983; Andreev 1984; Zabolotnikov (1984)). It should also be taken into account.

Unfortunately, we have measurements along the single trajectory within the stream (the Earth's orbit). If we had measurements made far from the Earth, their significance hardly could be overestimated.

\subsection{Bimodality of cometary streams}

Why do some profiles have two maxima, and some profiles have only one? The detailed explanation is given in (Ryabova 2001b) or (Ryabova 2001c). Briefly, the reason is that the orbital parameters of the meteoroids ejected from the parent comet when it approaches perihelion, differs from those of the particles ejected when the comet moves away from perihelion. This difference enhances in time, resulting in the formation of two layers (preand after-perihelion) in the stream. The location of the layers will be different for the meteoroids of different masses, mainly because of different ejection velocity, and also due to radiational effects. The Earth in its motion crosses these layers one after the other, we see two activity maxima (see panels A or B in fig. 1). But the layers for each differential stream have intersection along the reference (for this differential stream) orbit. So, if the Earth's way passes near, say, reference orbit $m_{3}$, the distance between layers is too small, and we see only one maximum for $m_{3}$-activity curve (e.g. panels $\mathrm{C}$ or $\mathrm{D}$ in fig. 1 ).

Results of the simulation show that a stream generated by cometary type during one revolution should produce a bimodal shower of specific type, where the distance in solar longitude between the first and the second maxima depends on the mass of meteoroids. 
The real Geminid shower possess the feature. So it is reasonable to suggest that it was being produced by Comet Phaethon.

But the flux curve is not the only parameter we could use. It is reasonable to suggest that above mentioned pre- and post-perihelion layers have more or less different orbital characteristics. And it is really so. At any rate it is so for the model (Ryabova 2001b, fig. 6; Ryabova 2004, fig. 3). The problem is that quantity and quality of observed meteoroid orbits are insufficient to use for comparison with the model. We need the observational data on the orbits that meet the following conditions (Ryabova 2001b; Ryabova 2001c). 1. The mass of meteoroids should be less than $10^{-3} \mathrm{~g}$ or, better still, $10^{-4} \mathrm{~g}$. Of course, the observations of meteoroids with fixed mass cannot be ensured; however, the mass range should be as narrow as possible, at least within the order of magnitude.

2. The accuracy of determination of orbital elements should be sufficiently high.

3. The number of orbits should be statistically sufficient for regression analysis.

And, again, we meet problem of observational selection. There are two approaches: to exclude observational selection factors from observations or to include them into the model. In the first case we can only outline limits of observability, but not restore missing observations. It could be useful and instructive to plan and carry out a special experiment. For example to do video observations of the Geminids, and to model the observations. Certainly, it is not a simple task.

\subsection{Some possible lines for research}

Besides those mentioned above there are several following interesting problems that could be solved.

1) To use radiant structure for calibration of the model. Certainly, the problem of the observational selection elimination should be the main difficulty.

2) To study influence of the Earth's passage through the stream on fine stream structure.

3) To model grouping in the stream. Data of observations exists (e.g. Karpov 2001; Karpov \& Gainullin (2002)).

4) To model the stream's size distribution. There are results of Singer \& Stanley (1980) regarding the flux of submicron particles $\left(m<10^{-15} \mathrm{~g}\right)$ observed in the Geminid stream on satellite Explorer 46 . Lifiteme for such small particles is about several years, so they are probably a result of collisions in the stream or with sporadical meteoroids.

5) To find a shape of Geminid's meteoroids from light-curve flickering (Beech 2002).

\section{Origin of the Arietid and Geminid type meteoroid streams}

The question of the Arietid and Geminid type meteoroid streams origin is still an open question. Twenty years ago Lebedinets $(1984,1985)$ noted that there are about 30 meteoroid streams in the catalogue of radar meteors by Lebedinets, Korpusov \& Sosnova (1972) with specific orbits: small size $(a \leqslant 2$ AU) and very small perihelion distance $(0.01 \leqslant q \leqslant 0.1 \mathrm{AU})$, including the Daytime Arietids $(q=0.08 \mathrm{AU}, a=2.1 \mathrm{AU})$. The orbits of another several tens of streams, including the Geminid stream, have the same small size, but larger perihelion distance $(0.1 \leqslant q \leqslant 0.2 \mathrm{AU})$.

The fact that a parent comet (as we discussed earlier, asteroid (3200) Phaethon is probably a dormant comet) was detected with confidence only for the Geminid stream is not surprising, because the lifetime of a comet in such orbits is short. But how can a comet appear on such orbits? It can not be transferred from a long-period orbit under the influence of a strong gravitational perturbation by Jupiter or Saturn, because its aphelion is far inside the Jupiter's orbit $\left(a_{J} \approx 5.2 \mathrm{AU}\right)$. The gradual shift of the aphelion from the 
orbit of Jupiter due to repeated weak perturbations is not possible, because the comet should extinct far in advance of generating of an Arietid-type stream. In principle, the transformation of typical cometary orbits in Arietid-type orbits is possible by a single perturbation from the Earth or Venus (Andreev, Terent'eva \& Bayuk 1990; Andreev, Terent'eva \& Bayuk 1991; Terent'eva \& Bayuk 1991). But what is the probability of such event? Is it high enough to form several tens of meteoroid streams?

One of the possible mechanism for the transformation of cometary orbits into Arietidtype orbits could be the reactive drag of the sublimating cometary nucleus. According to estimations of Lebedinets $(1984,1985)$, who modelled the transformation for a set of cometary orbits with perihelion distances $0.01 \leqslant q \leqslant 0.1 \mathrm{AU}$, aphelia near the orbit of Jupiter $(Q=5.2 \mathrm{AU})$, and nuclei radii $0.02 \leqslant R_{c} \leqslant 2 \mathrm{~km}$, decresing of aphelion distance from 5.2 AU till $4 \mathrm{AU}$ is quite possible in a time from half to several cometary periods.

The perihelion distance of a comet or a young compact swarm on the Arietid-type orbit is very sensitive to perturbations in aphelion, and can be changed by a weak single perturbation by Jupiter. For example, on changing the velocity in aphelion within $\pm 1 \mathrm{~km} \mathrm{~s}^{-1}$ the perihelion distance changes in the range $0.01-0.14 \mathrm{AU}$, and the aphelion distance changes only slightly.

In the case of strong reactive transformation of the cometary orbit, the resulting stream should be more wide, than the one produced under 'normal' conditions. It is so for the Geminid stream.

The described research was only a pilot one and, unfortunalely, was not continued.

Certainly, comparative studies of the Geminid and Arietid meteoroid streams could be helpful in clarifying their origin and formation.

The Arietids is a daytime stream in the Northern hemisphere, that is why it was undiscovered until beginning of radar observations of meteors $\dagger$. In the Southern hemisphere the shower can be observed visually (Jenniskens 1994). It is a wide stream $\left(\lambda_{\odot} \approx 68^{\circ}-95^{\circ}\right)$, with maximum at June 9 th and activity approximately equal to the Geminids or Quadrantids (Jenniskens 1994; Campbell-Brown 2004).

There were several attempts to identify a parent body for the stream. McIntosh (1990) based on similarities in behaviour of orbital elements suggested that Comet P/Machholtz 1986 VIII, Comet 1491 I, the Quadrantid, $\delta$-Aquarid and D.Arietid meteoroid streams have a common origin. Babadzhanov \& Obrubov (1992) using the method of filling volume, described above in Subsection 2.2, simulated the evolution of the Comet $\mathrm{P} /$ Machholtz meteoroid stream. The stream may produce eight meteor showers, including six known ones: the D.Arietids, Quadrantids, Ursids, Northern and Southern $\delta$-Aquarids, and $\alpha$-Cetids. The authors estimated the age of the stream in 7.5 millenia. But according to my own rough estimation, time for full revolution of the node for the Quadrantid stream is about 16 thousand years. At the same conference $\ddagger$ results of the Quadrantid meteoroid stream modelling (Jones \& Jones 1992) were presented. The stream consisting of 500 test particles was ejected 4000 years ago from the mean orbit of the Quadrantids. Considering quasi-constants of the motion the authors found that the Quadrantids and D.Arietids are unlikely to be members of a single complex. Later the same authors (Jones \& Jones 1993) made another simulation based on evolution of the Comet Machholtz and came to a conclusion that the comet probably gave birth to the Quadrantid, D.Arietid, Southern $\delta$-Aquarid meteoroid streams, but strong doubt about D.Arietids exists.

Two years ago results of two researches were published practically simultaneously. Gorbanev \& Knyaz'kova (2003) found that a genetic association probably exists for the

$\dagger$ A historical review can be found in Lovell (1954), Kronk (1988) or Campbell-Brown (2004). $\ddagger$ Asteroids, Comets and Meteors 1991 . 
meteoroid stream producing the D.Arietids and Southern $\delta$-Aquarids, and the Marsden comet group, discovered in 2002. Ohtsuka, Nakano \& Yoshikawa (2003) concluded that comet Machholtz, the D.Arietids, the Marsden group may be generally associated.

The first incident flux density curve for the D.Arietids was obtained recently by Campbell-Brown (2004). It is calculated with constant value for the mass distribution index $(s=2.1)$, so probably will be revised.

To summarize: The D.Arietid meteoroid stream still has no recognized parent body. We have several hypothesis, which need further investigations. Maybe it worth to search among newly-discivered Amor and Apollo asteroids? To construct a preliminary model of the D.Arietid meteoroid stream we need also an estimate of the age.

\section{The Perseid meteoroid stream}

There are only a few works devoted to the Pereseid modelling. Some of them (Wu \& Williams 1993; Williams \& Wu 1994; Harris \& Hughes 1995; Harris, Yau \& Hughes (1995)) are reviewed in the paper by Brown \& Jones (1998) discussed below in Subsections $7.1-7.3$.

\subsection{Model description}

The formation scheme for the Perseid meteoroid stream and, consequently, the structure of the stream is very different from those of the Geminid stream. The Perseid's parent comet $109 \mathrm{P} /$ Swift-Tuttle is still alive and adds meteoroids into the stream at every revolution. Brown \& Jones (1998) simulated ejection of particles at each perihelion passage of comet $109 \mathrm{P}$ from 59 to $1862 \mathrm{AD}$ and integrated orbits of the meteoroids (18.24 mln meteoroids in sum) till their descending nodes for times closest to 1992, i.e. the last perihelion passage of 109P. Four ejection velocity formulae were used: the first model was based on Crifo (1995) results, the other three are based on Jones (1995) modifications of Whipple's formula. It should be noted that the models of ejection velocities are different, but not dramatically different.

The ejection velocity depends on particle windage, $A / m$. In the discussed work spherical particles with three densities were used, namely $0.1,0.8$, and $4.0 \mathrm{~g} \mathrm{~cm}^{-3}$. In total we have 12 distinct models. For each 10000 test meteoroids were ejected at different masses from interval $10^{-5}-10 \mathrm{~g}$ for each perihelion passage of $109 \mathrm{P} /$ Swift-Tuttle.

\subsection{Age of the stream}

To estimate age of the stream long-term integrations was also performed over the interval from 5 to 100 thousands years ago using the SWIFT symplectic integrator. At first to take into account errors in initial conditions, 20 clone orbits were integrated from 1862 backward for 100000 years. Then the two model streams were generated, using the two 'extreme' orbits, and integrated forward. An attempt to estimate age of the stream using the radiant size of the Perseid shower, the photographic radiant locations at maximum activity, the rate of change in the apparent location of the maximum, the width of the ZHR profile gave very different ages in the interval $(7-180) \times 10^{3}$ years. But the long duration of the Perseid shower implies a lower limit for the age of the stream of the order of $10^{5}$ years.

It is interesting that according to the results of modelling some Perseid meteoroids may encounter the Earth at their ascending nodes in mid-March. The Gamma Normids and the Theta Centaurids are the candidates for twin showers of the Perseids. If the existence of streams will be confirmed, that means a stream age of at least 50-75 thousands years. 
Babadzhanov \& Obrubov (1987) did not consider the Perseid stream. As it was mentioned in Subsection 2.2, my rough estimate has shown that the Perseid stream could produce to 8 showers during $1 \mathrm{mln}$ years. Maybe it worth to check?

\subsection{Models and observations}

From the analysis of the results, the authors have drawn several conclusions regarding role of initial conditions and effects of gravitational and radiational perturbations, and density assumed for meteoroids. I will not retell the conclusions, preferring enlarge on fitting models to observations.

What observational data may be used as criterions for validation of the Perseid models? The main "anchor" is the shower activity, especially locations and strength of outburst peaks. The secondary one is the geocentric radiant distribution. Unfortunately, the present size of the measurement errors makes orbital elements insuitable for this purpose. Data on mass distribution index in the Perseids exist, but they were not used in the research. So, it is a reserve for the future.

The best fit to the observed outbursts from the years 1989-1996 were found from the model using Jones (1995) modification of Whipple's formula with $r^{-0.5}$ dependence and meteoroid densities between 0.1 and $0.8 \mathrm{~g} \mathrm{~cm}^{-3}$.

The locations and strength of the observed visual peaks associated with the outburst component of the stream and the model one are in good agreement, besides the 1993 and 1994 peak locations, and the 1994 - 1995 peak strength, which were underestimated in the models. As it turned out in 1993 ejections from 1862 and 1610 are predominant in meteoroid population. In 1994 the main contributions are from 1862 and 1479, and in 1995 - from 1479 and 1862. No observations exists for returns of 1479 and 1610, but for 1862 approach there are detailed observations (Sekanina 1981; Fomenkova, Jones, Pina, et al. 1995).

It worth to note that activity of the model comet was taken as constant, and ejection velocity vectors were taken isotropically distributed within the sunward hemisphere. There are two possible approaches to fit a model to observations. The direct approach is to change the model, say, to increase the number of ejected particles, until a coincidence with observations of the shower. This approach requires new calculations. Another, the indirect approach, mentioned in Subsection 2.2 as weighting coefficients technique, consist in giving a weight to some model meteoroids contributing in the peak to change it to favourable location or strength, and to analyze coincidence with observations of the comet dust production.

For example, the authors of the model mentioned that it is possible to change the mean nodal longitude of the 1993 and 1994 peaks implying that almost all ejections had a strong component normal to the cometary plane in the north direction. It could be reasonable to look closer at the model meteoroids having "correct" nodal longitudes (certainly, if such meteoroids exist). Maybe their ejection velocity vectors are within registered jets?

In my opinion this model is the best model for the Perseid stream that could be done with existing observational data. Let us imagine, that in the coming years a flux profile of a high resolution together with the profile for mass distribution index $s$ will be obtaided from radar observations of the Perseids. Then, this model could serve once more, if the authors will consider it necessary, and if the files with information will not be lost. 


\section{The Leonid meteoroid stream}

In a sense, the theory of meteoroid streams began from strong Leonid meteor storms of 1799 and 1833. Detailes can be found elsewhere (Lovell 1954; Wu \& Williams 1996; Brown 1999). The parent comet of the Leonids is Comet 55P/ Tempel-Tuttle, and storms occur in years of its return to the Sun. In other time, i.e. when the comet is far from perihelion, activity of the Leonids is very low. That is why it had been generally accepted that the Leonid meteoroid stream is young, and the meteoroids had no enough time to spread along the orbit, till Williams (1997) proposed very a interesting explanation of the facts. Gravitational perturbations from planet Uranus, which is on a 5:2 mean motion resonance with the Leonid parent comet, "sweep out" the meteoroids from all the stream orbit except small arc located near the nucleus of the comet.

For a long time efforts of "streams modellers" were concentrated on the Leonids predictions. One of the first advantageous attempt was made by Kazimirčak-Polonskaya, Belyaev, Astapovič \& Terent'eva (1968). Besides, it was one of the first meteoroid stream's models. The authors numerically integrated orbits of 17 model meteoroids, and found time of maximum activity for the Leonids of 1966 with accuracy about $1 \mathrm{hr}$.

The increase in the activity of the Leonids from 1998 to 2002 stimulated many researches to model the Leonid stream with the main aim to predict showers (Brown \& Jones 1996; McNaught \& Asher 1999; Lyytinen \& Van Flandern 2000; Brown \& Cooke 2001 etc.). But till now the Leonid stream as a whole was not studied. The last Leonid stream model is already near to that, but still is aimed at predictions. The full description of the method and results are presented in (Vaubaillon, Colas \& Jorda 2005a,b), but a brief of the model could be also found in (Vaubaillon \& Colas 2005; Vaubaillon, Lyytinen, Nissinen \& Asher 2003; Vaubaillon \& Colas 2002; Vaubaillon 2002).

The authors apply quite classic approach to meteoroid stream modelling. Using the definite model of the meteoroid ejection from the parent comet, the ejection of $2.5 \times$ $10^{5}$ particles was modelled for 29 perihelion returns of comet $55 \mathrm{P}$. The dates of perihelion passages are from 1300 to 1998, from 604 to 802, and 1001. The particle's size range 0.1-100 mm was divided into 5 bins. Evolution of the test particles was calculated till the present time, taking into account gravitational and non-gravitational forces. Then, from all model meteoroids there were selected those with nodes close to the Earth, and their parameters were analyzed. The comparison between predictions and observations for the time of maximum activity shown a good agreement with a difference till a few tens of minutes. The greatest differences are found for years 1999 and 2000. Authors assumed, that another still-unknown stream had been encountered. But certainly other explanations are possible. For example, Brown \& Jones (1998) have shown how time of maximum can be shifted by some corrections in initial parameters.

$A$ resume: this model has rather large potential for further study.

\section{Resonances in meteoroid streams}

Resonances in meteoroid streams is a topic worthy of a separate review. Here I'd like to dwell on some works showing that mean motion resonances can cause evident changes in the structure of a meteoroid stream. Emel'yanenko (2001) also noted that resonant streams are important objects for ground observations. These structures have well determined parameters if the mean motion commensurabilities are known. This can be used to improve physical models of meteor phenomenon.

Investigating the Quadrantid meteoroid stream (more precisely, the part of the stream close to the 2/1 resonance with Jupiter) by Shubart's (1978) averaging method, Froeschlé 
\& Scholl (1986) found that the stream breaks up into arcs of different sizes with distinctly different dynamical evolution. The other seven meteoroid streams (June Bootids, Annual Andromedids, Librids, June Lyrids, July Phoenicids, Pegasids, and December Phoenicids) known to be located in mean motion resonances with Jupiter do not reveal the same kind of splitting into arcs (Scholl \& Froeschlé 1988).

The existence of gaps in the distribution of the semimajor axes of meteoroids in the Perseid meteoroid stream was found by Wu \& Williams (1995). The gaps are coincided with the location of low-order mean motion resonances with the major planets. Wu \& Williams carried out a numerical integration of 11000 test meteoroids over $1000 \mathrm{yr}$. The initial orbital elements of the sample were randomly chosen from a range near the value of the same element of $\mathrm{P} /$ Swift-Tuttle. These integrations showed that gaps in $1 / a$ distribution formed at some of the mean motion resonance locations in a very short interval of $150 \mathrm{yr}$.

Later the results of Wu \& Williams (1995) were discussed by Murray (1996). Murray has shown that the gaps in orbital element distributions of asteroid families and meteoroid streams can be produced by the genuine removal of objects by resonant effects ('real' gaps) and those that could result from a failure to identify some material as belonging to a particular group because of the resonant perturbations ('imaginary' gaps). It was also shown that some gaps in the distribution of the reciprocal semimajor axes of Perseid meteor orbits for visual meteors discussed by Wu \& Williams (1995) probably are imaginary. As to results of numerical modelling by $\mathrm{Wu} \&$ Williams, most of the gaps can be regarded as real ones but the reason for the removal of meteoroids is debatable. It could be both resonant effects and direct perturbation due to a close approach to a planet. So, further studies should be carried out. Murray (1996) also stressed the role of observational selection effect for the orbital data of meteoroids.

A young meteoroid stream generated during an approach of its parent comet to the Sun begins to disperse, as a rule. However librating meteoroids can produce long-lived highdensity substreams. This is important from practical point of view: hazard to spacecrafts.

Asher \& Clube (1993) described a model of a meteoroid swarm in the Taurid Complex and in $7 / 2$ resonance with Jupiter. Later Asher \& Izumi (1998) made a stronger test of the model using meteor observations of the Nippon Meteor Society over the past six decades. At high statistically significant level a single, dominant concentration of meteoroids in the stream can explain all (to date) observed years of enhancement.

Leonid meteor shower of 1998 surprised astronomers by unexpected high incidence of bright meteors about $16 \mathrm{~h}$ before the predicted maximum, i.e. in 1998 November 17.1. Asher, Bailey \& Emel'yanenko (1999) have noted that the observed fireball outburst was distinguished by a predominance of very bright meteors originating from large dust grains with sizes ranging up to a few centimeters. The highest level of fireball activity lasted approximately half a day, indicating the presence of a narrow concentrated substream. Investigation have shown that the outburst is explained by the ejection of meteoroids into the 5/14 mean motion resonance with Jupiter mainly during the perihelion passage of Comet 55P/Tempel-Tuttle in 1333. The meteoroids could not spread around the orbit in a usual way. Instead they produced a concentrated swarm, taking the form of an arc of material close to, but not necessarily identical with, the parent cometary orbit.

It could be extremely interesting to study possible high-concentrated substreams in a full model (like described above the Geminid and the Perseid stream models) of a stream, considering space location of the swarms, and their evolution. As the first candidates for such study I see the Quadrantid and the Leonid meteoroid streams. The Geminid stream also has two very narrow zones located in the mean motion resonances $7 / 1$ and $8 / 1$ 
with Jupiter (Emel'yanenko 2001), so it is desirable to look closer at the behavior of meteoroids in these locations.

\section{Acknowledgements}

The author would like to acknowledge the financial support from the Organizers, which make it possible her attendance at the symposium and consequently stimulated the writing of this review.

\section{References}

Andreev, G.V. 1984, Solar System Research 18, 158

Andreev, G.V. 1986, Astronomiya i geodeziya 15, 164 (in Russian)

Andreev, G.V. 1987, Handbook for MAP 25, 305

Andreev, G.V., Terent'eva, A.K., \& Bayuk O.A. 1990, in: C.-I.Lagerkvist, H.Rickman, B.A.Lindblad \& M.Lindgren (eds.), Asteroids, Comets, Meteors III, (Uppsala universitet Reprocentralen HSC, Uppsala), p. 493

Andreev, G.V., Terent'eva, A.K., \& Bayuk O.A 1991, Astronomich. Vestnik 25, 177 (in Russian) Andreev, G.V. 1995, Astronomicheskij vestnik 29, 151 (in Russian)

Arter, T.R., \& Williams I.P. 2002, Mon. Not. R. Astron. Soc. 329, 175

Asher, D.J. \& Clube, S.V.M. 1993, QJRAS 34, 481

Asher, D.J. \& Izumi, K. 1998, Mon. Not. R. Astron. Soc. 297, 23

Asher, D.J., Bailey, M.E., \& Emel'yanenko, V.V. 1999, Mon. Not. R. Astron. Soc. 304, L53

Asher, D.J. 1999, Mon. Not. R. Astron. Soc. 307, 919

Babadzhanov, P.B., Zausaev, A.F., \& Obrubov, Yu.V. 1980, Bull. Inst. Astrophys. of TadzhSSR 69-70, 45 (in Russian)

Babadzhanov, P.B. \& Obrubov, Yu.V. 1987, in: Z.Ceplecha \& P. Pecina (eds.), Interplanetary Matter, Publ. Astron. Inst. Czechosl. Acad. Sci., Vol. 67(2), (Ond(r)ejov: Czechosl. Acad. Sci.), p. 141

Babadzhanov, P.B. \& Obrubov, Yu.V. 1992, in: A.Harris \& E.Bowell (eds.), Proc. Asteroids, Comets and Meteors 1991, (Lunar and Planetary Inst., Flagstaff, AZ), p. 27

Beech, M. 2002, Mon. Not. R. Astron. Soc. 336, 559

Bel'kovich, O.I. 1983, Sol. Syst. Res. 17, 83

Bel'kovich, O.I. 2005, private communication

Bordovitsyna, T., Avdyushev, V., \& Chernitsov, A. 2001, Cel. Mech. Dyn. Astron. 80, 227

Bronshten, V.A. 1983, Sol. Syst. Res. 17, 175

Brown, P. \& Jones, J. 1996, in: B.A.S.Gustafson, M.Hanner (eds.), Physics, Chemistry, and Dynamics of Interplanetary Dust, Proc. IAU Coll. No.150 (ASP 104) (San Francisco: Astron. Soc. of the Pasific), p. 113

Brown, P. \& Jones, J. 1998, Icarus 133, 36

Brown, P. 1999, Icarus 138, 287

Brown, P. \& Cooke, B. 2001, Mon. Not. R. Astron. Soc. 326, L19

Campbell-Brown, M. 2004, Mon. Not. R. Astron. Soc. 352, 1421

Crifo, J.F. 1995, ApJ 445, 470

Crifo, J.F. \& Rodionov, A.V. 1997, Icarus 127, 319

Dohnanyi, D. 1970, J. Geophys. Res. 75, 3468

Dohnanyi, D. 1978, in: J.A.M. McDonnell (ed.), Cosmic dust, (Chichester: Wiley-Interscience), p. 527

Emel'yanenko, V.V. 2001, in: B.Warmbein (ed.), Proc. Meteoroids 2001 Conf., ESA SP-495 (Noordwijk: European Space Agency), p. 43

Fomenkova, M., Jones, B., Pina, R., Puetter, R., Sarmecanic, J., Gehrz, R., \& Jones, T. 1995, AJ 110,1866

Fox, K., Williams, I.P., \& Hughes, D.W. 1982, Mon. Not. R. Astron. Soc. 199, 313

Fox, K., Williams, I.P., \& Hughes, D.W. 1983, Mon. Not. R. Astron. Soc. 205, 1155

Froeschlé, C. \& Scholl, H. 1986, Astron. Astrophys. 158, 259 
Gockel, C. \& Jehn, R. 2001, Mon. Not. R. Astron. Soc. 317, L1

Gorbanev, Y.M. \& Knyaz'kova, E.F. 2003, Sol. Syst. Res. 37, 506

Hamid, S.E. 1951, AJ 56, 126

Harris, N.W. \& Hughes, D.W. 1995, Mon. Not. R. Astron. Soc. 273, 992

Harris, N.W., Yau, K.C.C., \& Hughes, D.W. 1995, Mon. Not. R. Astron. Soc. 273, 999

Hughes, D.W. 2000, Planet. Space. Sci. 48, 1

Jenniskens, P 1994, Astron. Astrophys. 287, 990

Jenniskens, P 2001, WGN, Journal of the International Meteor Organization 29, 165

Jenniskens, P 2002, in: B.Warmbein (ed.), Proc. Asteroids, Comets, Meteors - ACM2002 Conf., ESA SP-500 (Noordwijk: European Space Agency), p. 117

Jones, J. 1985, Mon. Not. R. Astron. Soc. 217, 523

Jones, J. \& Hawkes, R.L. 1986, Mon. Not. R. Astron. Soc. 223, 479

Jones, J. \& Jones, W. 1992, in: A.Harris \& E.Bowell (eds.), Proc. Asteroids, Comets and Meteors 1991, (Lunar and Planetary Inst., Flagstaff, AZ), p. 269

Jones, J. \& Jones, W. 1993, Mon. Not. R. Astron. Soc. 261, 605

Jones, J. 1995, Mon. Not. R. Astron. Soc. 275, 773

Karpov, A. 2001, in: B.Warmbein (ed.), Proc. Meteoroids 2001 Conf., ESA SP-495 (Noordwijk: European Space Agency), p. 27

Karpov, A. \& Gainullin R. 2002, in: B.Warmbein (ed.), Proc. Asteroids, Comets, Meteors ACM2002 Conf., ESA SP-500 (Noordwijk: European Space Agency), p. 249

Kazimirčak-Polonskaya, E.I., Belyaev, N.A., Astapovič, I.S., \& Terent'eva, A.K. 1968, in: L.Kresák \& P.M.Millman (eds.), Physics and Dynamics of Meteors, Proc. IAU Symp. No.33, (Dordrecht, Reidel), p. 449

Kazimirchak-Polonskaya, E.I., Belyaev, N.A., \& Terent'eva, A.K. 1972, in: G.A.Chebotarev, E.I. Kazimirchak-Polonskaya \& B.A. Marsden (eds.), The Motion, Evolution of Orbits, and Origin of Comets, Proc. IAU Symp. No.45, (Dordrecht, Reidel), p. 462

Kronk, G.W. 1988, Meteor showers: A descriptive catalog, (Enslaw Publishers, New Jersey)

Lebedinets, V.N., Korpusov, V.N., \& Sosnova A.K. 1972, Trudy Inst. Eksp. Meteorol. 1(34), 88 (in Russian)

Lebedinets, V.N. 1984, Trudy Inst. Eksp. Meteorol. 14(110), 88 (in Russian)

Lebedinets, V.N. 1985, Sol. Syst. Res. 19, 101

Leinert, C., Röser, S., \& Buitrago, J. 1983, Astron. Astrophys. 118, 345

Lidov, M.L. 1961, Iskusstvennye sputniki Zemli 8, 5 (in Russian)

Lovell, A.C.B. 1954, Meteor astronomy, (Oxford Clarendon Press)

Lyytinen, E.J. \& Van Flandern, T. 2000, Earth, Moon, \& Planets 82, 149

Ma, Y., He, Y., \& Williams I.P. 2001, Mon. Not. R. Astron. Soc. 325, 379

Ma, Y., \& Williams I.P. 2001, Mon. Not. R. Astron. Soc. 325, 457

McIntosh, B.A. \& Jones, J. 1988, Mon. Not. R. Astron. Soc. 235, 673

McIntosh, B.A. 1990, Icarus 86, 299

McNaught, R.H. \& Asher, D.J. 1999, WGN, Journal of the International Meteor Organization 27,85

Moiseev, N.D. 1945, Trudy Gos. Astron. Inst. Moscov. Univ. 15, 75 (in Russian)

Murray, C.D. 1982, Icarus 49, 125

Murray, C.D. 1996, Mon. Not. R. Astron. Soc. 279, 978

Ohtsuka, K., Nakano, S., \& Yoshikawa, M. 2003, Publ. Astron. Soc. Japan 55, 321

Olsson-Steel, D. 1986, Mon. Not. R. Astron. Soc. 219, 47

Plavec, M. 1950, Nature 165, 362

Plavec, M. 1955, Bull. Astron. Inst. Czech. 6, 20

Plavec, M. 1955, in: T.R. Kaiser (ed.), Meteors, Proc. Second Intern. Symp. Phys. Meteors., p. 168

Plavec, M. 1957, On the origin and early stages of the meteoroid streams (Praha)

Richter, K., Curdt, W., \& Keller, H.U. 1991, Astron. Astrophys 250, 548

Ryabova, G.O. 1989, Sol. Syst. Res. 23, 158

Ryabova, G.O. 1997, Sol. Syst. Res. 31, 277

Ryabova, G.O. 1999, Sol. Syst. Res. 33, 224 
Ryabova, G.O. 2001a, in: B.Warmbein (ed.), Proc. Meteoroids 2001 Conf., ESA SP-495 (Noordwijk: European Space Agency), p. 63

Ryabova, G.O. 2001b, in: B.Warmbein (ed.), Proc. Meteoroids 2001 Conf., ESA SP-495 (Noordwijk: European Space Agency), p. 77

Ryabova, G.O. 2001c, Sol. Syst. Res. 35, 151

Ryabova, G.O. 2003, Mon. Not. R. Astron. Soc. 341, 739

Ryabova, G.O. 2004, in: M.Triglav-Čekada \& C.Trayner (eds.), Proc. Intern. Meteor Conf. 2003, (Internat. Meteor. Org.), p. 131

Ryabova, G.O. 2005, in: Z.Knezevic \& A.Milani (eds.), Dynamics of Populations of Planetary Systems, Proc. IAU Coll. 197, (Cambridge University Press), p. 411

Scholl, H. \& Froeschlé, C. 1988, Astron. Astrophys. 195, 345

Schubart, J. 1978, in: V.Szebehely (ed.), Dynamics of Planets and Satellites and Theories of their Motion, (Reidel, Dordrecht), p. 173

Sekanina, Z. 1981, AJ 86, 1741

Singer, S.F. \& Stanley, J.E. 1980, in: I.Halliday \& B.A.McIntosh (eds.), Solid Particles in the Solar System, Proc. IAU Symp. 90, (Reidel, Dordrecht), p. 329

Steel, D.I. \& Elford, W.G. 1986, Mon. Not. R. Astron. Soc. 218, 185

Terent'eva, A.K. \& Bayuk O.A 1991, Bull. Astron. Inst. Czechosl. 42, 377

Tokhtas'ev, V.S. 1982, in: O.I.Bel'kovich, P.B.Babadzhanov, V.A.Bronshten \& N.I.Suleimanov (eds.), Meteor Matter in the Interplanetary Space, (Moscow-Kazan) p. 162

Vaubaillon, J. 2002, WGN, Journal of the International Meteor Organization 30, 144

Vaubaillon, J. \& Colas, F. 2002, Proc. Asteroids, Comets, Meteors - ACM2002 Conf., ESA SP-500 (Noordwijk: European Space Agency), p. 181

Vaubaillon, J., Lyytinen, E., Nissinen, M., \& Asher, D. 2003, WGN, Journal of the International Meteor Organization 31, 131

Vaubaillon, J. \& Colas, F. 2005, Astron. Astrophys. 431, 1139

Vaubaillon, J., Colas, F., \& Jorda L. 2005a, Astron. Astrophys. 439, 751

Vaubaillon, J., Colas, F., \& Jorda L. 2005b, Astron. Astrophys. 439, 761

Weiss, E. 1868, Astronomische Nachrichten 72, 81

Whipple, F. 1951, ApJ 113, 464

Whipple, F. 1967a, Smithsonian Astrophys. Obs. Spec. Rept. 239, 1

Whipple, F. 1967b, in: J. Weinberg (ed.), The Zodiacal Light and the Interplanetary Medium, NASA SP-150 (Washington: Scientific and Technical Information Division, National Aeronautics and Space Administration), p. 409

Williams, I.P., Murray, C.D., \& Hughes, D.W. 1979, Mon. Not. R. Astron. Soc. 189, 483

Williams, I.P. \& Wu, Z. 1994, Mon. Not. R. Astron. Soc. 269, 524

Williams, I.P. 1996, Earth, Moon, E Planets 72, 231

Williams, I.P. 1997, Mon. Not. R. Astron. Soc. 292, L37

Williams, I.P. 2001, in: B.Warmbein (ed.), Proc. Meteoroids 2001 Conf., ESA SP-495 (Noordwijk: European Space Agency), p. 33

Wu, Z., \& Williams, I.P. 1993, Mon. Not. R. Astron. Soc. 264, 980

Wu, Z., \& Williams, I.P. 1995, Mon. Not. R. Astron. Soc. 276, 1017

Wu, Z., \& Williams, I.P. 1996, Mon. Not. R. Astron. Soc. 280, 1210

Zabolotnikov, V.S. 1984, Solar System Research 18, 35 